\title{
Modal Logic Axioms Valid in Quotient Spaces of Finite CW-Complexes and Other Families of Topological Spaces
}

\author{
Maria Nogin and Bing Xu \\ Department of Mathematics, California State University, Fresno, CA, USA \\ Correspondence should be addressed to Maria Nogin; mnogin@csufresno.edu
}

Received 24 August 2016; Accepted 8 December 2016

Academic Editor: Ram U. Verma

Copyright (C) 2016 M. Nogin and B. Xu. This is an open access article distributed under the Creative Commons Attribution License, which permits unrestricted use, distribution, and reproduction in any medium, provided the original work is properly cited.

In this paper we consider the topological interpretations of $\mathscr{L}_{\square}$, the classical logic extended by a "box" operator $\square$ interpreted as interior. We present extensions of $\mathbf{S} \mathbf{4}$ that are sound over some families of topological spaces, including particular point topological spaces, excluded point topological spaces, and quotient spaces of finite CW-complexes.

\section{Introduction}

It was shown by McKinsey and Tarski in [1] that every finite well-connected topological space is an open image of a metric separable dense-in-itself space. This implies that the modal logic S4 is complete in any metric separable dense-in-itself space, for example, $\mathbb{R}^{n}$. The original proof of McKinsey and Tarski was quite tedious and technical; simpler, shorter, more geometric proofs were given in [2-5]. It follows that $\mathbf{S 4}$ is sound and complete over all topological spaces; that is, a formula is derivable from the axiom system of $\mathbf{S 4}$ if and only if it is valid in all topological spaces. Note that if a formula is valid in some topological space, it does not follow that this formula is derivable from S4.

In Section 2 we recall all necessary definitions, and in Section 3 we consider two axioms, denoted by $M$ and $G$, each not derivable from $\mathbf{S} 4$ and the other one, and for each of them we give necessary and sufficient conditions under which it is valid in a quotient space of a finite CW-complex, a particular point topological space, and an excluded point topological space.

\section{Preliminaries}

2.1. CW-Complexes and Their Quotient Spaces, Particular Point Topological Spaces, and Excluded Point Topological Spaces

Definition 1. Let $X$ be a CW-complex. Its quotient space $Q(X)$ is a topological space whose points are in one-to-one correspondence with cells of $X$, and a subset of $Q(X)$ is open if and only if the union of the corresponding cells is open in $X$.

Definition 2. If $X$ is a CW-complex, its cell $c$ is called a top cell if it is not in the boundary of any other cell.

Remark 3. A single point in $Q(X)$ is open if and only if it corresponds to a top cell.

Definition 4. Let $X$ be any nonempty set and $p \in X$. Collection $T_{p}=\{S \subseteq X \mid p \in S$ or $S=\emptyset\}$ of subsets of $X$ is called the particular point topology on $X([6, \mathrm{p} .44])$.

Remark 5. Since, for any particular point topological space $X$ with the particular point $p \in X$, any nonempty open subset includes $p$, it follows that the only closed subset that includes $p$ is $X$.

Definition 6. Let $X$ be any nonempty set and $e \in X$. Collection $T_{e}=\{S \subseteq X \mid e \notin S$ or $S=X\}$ of subsets of $X$ is called the excluded point topology on $X$ ([6, p. 47]).

Remark 7. Since, for any excluded point topological space $X$ with the excluded point $e \in X$, every proper open subset of $X$ excludes $e$, it follows that every nonempty closed set includes e.

Example 8. The quotient space of a bouquet of spheres (of any positive dimension) has excluded point topology. 


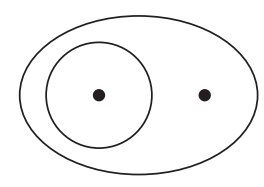

FIGURE 1

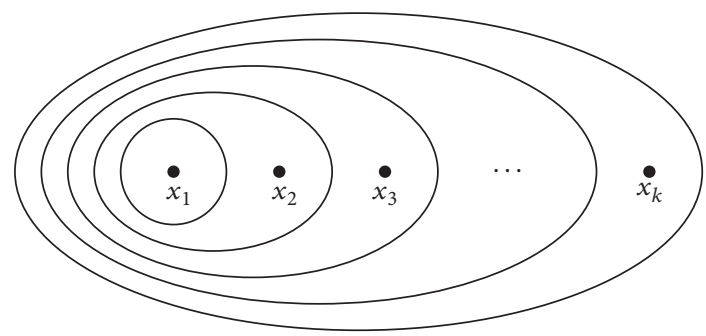

FIGURE 2

Example 9. Sierpiński space, a topological space with two points only one of which is open ([6, p. 44]), is a topological space with both particular point and excluded point topology. It is the quotient space of the standard CW-complex for $\mathbb{R} P^{1}$ (real projective plane) (see Figure 1).

Example 10. More generally, for each $n \in \mathbb{N}$, let $X_{n}=$ $\left\{x_{1}, x_{2}, \ldots, x_{n}\right\}$ with topology (see Figure 2).

$$
\begin{aligned}
T_{s} & =\left\{S \subseteq X \mid S=\emptyset \text { or } S=\left\{x_{1}, x_{2}, \ldots, x_{k}\right\} \text { for } 1 \leq k\right. \\
& \leq n\} .
\end{aligned}
$$

For each $n \in \mathbb{N}, X_{n}$ with topology $T_{s}$ is the quotient space of the standard CW-complex for $\mathbb{R} P^{n-1}$.

Remark 11. Topology $T_{s}$ is a subtopology of both a particular point topology (with the particular point being $x_{1}$ ) and an excluded point topology (with the excluded point being $x_{n}$ ).

\subsection{Modal Logic Language $\mathscr{L}_{\square}$ and Its Interpretation in Topological Spaces}

Definition 12. Let $\mathscr{L}_{\square}$ be a modal logic language consisting of propositional variables, conjunction $\wedge$, disjunction $\vee$, negation $\neg$, and modality $\square$. Modality $\diamond$ is defined as the dual to $\square$; that is, $\diamond P=\neg \square \neg P$.

Definition 13. S4 denotes the subset of $\mathscr{L}_{\square}$ consisting of all formulas that can be derived from the following axioms:

(i) All axioms of the classical propositional logic

(ii) $\square(A \rightarrow B) \rightarrow(\square A \rightarrow \square B)$

(iii) $\square A \rightarrow A$

(iv) $\square A \rightarrow \square \square A$

using the following rules:

(i) $(A, A \rightarrow B) / B$ (Modus ponens)

(ii) $A / \square A$ (necessitation).
Definition 14. A topological model of $\mathscr{L}_{\square}$ is a pair $\langle X,\|\cdot\|\rangle$, where

(1) $X$ is a topological space

(2) $\|\cdot\|$ is a valuation function mapping formulas in $\mathscr{L}_{\square}$ to subsets of $X$. It assigns each propositional variable $P$ an arbitrary subset $\|P\| \subseteq X$ and satisfies the following conditions for every $F, G \in \mathscr{L}_{\square}$ :

$$
\begin{aligned}
\|F \wedge G\| & =\|F\| \cap\|G\|, \\
\|F \vee G\| & =\|F\| \cup\|G\|, \\
\|\neg F\| & =\|F\|^{C}=X \backslash\|F\|, \\
\|\square F\| & =\operatorname{int}(\|F\|),
\end{aligned}
$$

where $\operatorname{int}(\cdot)$ is the topological interior operator.

Remark 15. Note that the modality $\diamond$ then maps to the topological closure operator; that is, $\|\triangleright F\|=\operatorname{cl}(\|F\|)$.

Definition 16. Let $F \in \mathscr{L}_{\square}$ be any formula. One says that $F$ is valid in a topological space $X$ if, for any topological model $\langle X,\|\cdot\|\rangle$, one has $\|F\|=X$.

Theorem 17 (topological completeness of S4, [1-5]). For any formula $F \in \mathscr{L}_{\square}$, the following two statements are equivalent:

(i) $F$ is derivable in $\mathbf{S} \mathbf{4}$.

(ii) $F$ is valid in all topological spaces.

\section{Axioms $M$ and $G$}

Consider the following two axioms (the first form of each axiom is the one appearing most often in the literature, but the second one will be more convenient for us):

$$
\begin{gathered}
M:(\square \diamond P) \longrightarrow(\diamond \square \neg P) \equiv(\diamond \square P) \vee(\diamond \square \neg P), \\
G:(\diamond \square P) \longrightarrow(\square \diamond \neg P) \equiv(\square \diamond P) \vee(\square \diamond \neg P) .
\end{gathered}
$$

Theorem 18. Both axioms $M$ and $G$ are valid in any particular point topological space.

Proof. Let $X$ be a particular point topological space with particular point $p$, and let $\|P\|=A$ be any subset of $X$.

Case $1(p \in A)$. Then $A$ is open. So $\operatorname{int}(A)=A$. Since the only closed set that includes $p$ is $X, \operatorname{cl}(\operatorname{int}(A))=\operatorname{cl}(A)=X$. Therefore $\|(\diamond \square P) \vee(\diamond \square \neg P)\|=\operatorname{cl}(\operatorname{int}(A)) \cup \operatorname{cl}\left(\operatorname{int}\left(A^{C}\right)\right)=X$.

Also, since the only closed set that includes $p$ is $X, \operatorname{cl}(A)=$ $X$. Then $\operatorname{int}(\operatorname{cl}(A))=\operatorname{int}(X)=X$. So $\|(\square \diamond P) \vee(\square \diamond \neg P)\|=$ $\operatorname{int}(\operatorname{cl}(A)) \cup \operatorname{int}\left(\operatorname{cl}\left(A^{C}\right)\right)=X$.

Case $2(p \notin A)$. Then $p \in A^{C}$. The rest is similar to case 1 , with the roles of $A$ and $A^{C}$ switched.

Theorem 19. (1) Axiom $M$ is valid in any excluded point topological space.

(2) Axiom $G$ is valid in an excluded point topological space if and only if the space has only 1 or 2 points. 
Proof. (1) Let $X$ be an excluded point topological space with excluded point $e$, and let $\|P\|=A$ be any subset of $X$.

Case $1(A=X)$. Then $\mathrm{cl}(\operatorname{int}(A))=\mathrm{cl}(\operatorname{int}(X))=X$.

Case $2(A=\emptyset)$. Then $A^{C}=X$, so $\operatorname{cl}\left(\operatorname{int}\left(A^{C}\right)\right)=\operatorname{cl}(\operatorname{int}(X))=$ $X$.

Case $3(A=X \backslash\{e\}, A \neq \emptyset)$. Then $\operatorname{cl}(\operatorname{int}(A))=\operatorname{cl}(A)=X$.

Case $4(A=\{e\}, A \neq X)$. Then $A^{C}=X \backslash\{e\}, A^{C} \neq \emptyset$; thus $\operatorname{cl}\left(\operatorname{int}\left(A^{C}\right)\right)=X$.

Case 5 (e $\in A \subset X$ and $A \neq\{e\})$. Then $\operatorname{cl}(\operatorname{int}(A))=\operatorname{cl}(A \backslash$ $\{e\})=A$ and $\operatorname{cl}\left(\operatorname{int}\left(A^{C}\right)\right)=\operatorname{cl}\left(A^{C}\right)=A^{C} \cup\{e\}$.

Case $6(e \notin A, A \neq \emptyset$, and $A \neq X \backslash\{e\})$. Then $e \in A^{C} \subset X$ and $A^{C} \neq\{e\}$. Thus this case is similar to case 5 , with the roles of $A$ and $A^{C}$ switched.

In each of these cases, we have

$$
\|(\diamond \square P) \vee(\diamond \square \neg P)\|=\operatorname{cl}(\operatorname{int}(A)) \cup \operatorname{cl}\left(\operatorname{int}\left(A^{C}\right)\right)=X \text {. }
$$

(2) An excluded point topological space with only one or two points is also a particular point topological space; thus axiom $G$ is valid by Theorem 18. If there are at least three points, let $e$ be the excluded point and let $a$ be any other point. Consider $A=\{a, e\}$. Then $\operatorname{int}(\operatorname{cl}(A))=\operatorname{int}(\operatorname{cl}(\{a, e\}))=$ $\operatorname{int}(\{a, e\})=\{a\}$ and $\operatorname{int}\left(\operatorname{cl}\left(A^{C}\right)\right)=\operatorname{int}(\operatorname{cl}(X \backslash\{a, e\}))=$ $\operatorname{int}(X \backslash\{a\})=X \backslash\{a, e\}$, so $\|(\square \diamond P) \vee(\square \diamond \neg P)\|=\operatorname{int}(\operatorname{cl}(A)) \cup$ $\operatorname{int}\left(\operatorname{cl}\left(A^{C}\right)\right)=X \backslash\{e\} \neq X$.

Remark 20. It is known (see, e.g., [7]) that axioms $M$ and $G$ are independent from each other and S4. Theorem 19 provides another proof of independency of $G$ from $\mathbf{S 4}+$ Axiom $M$, traditionally denoted by $\mathbf{S 4 . 1}$.

Theorem 21. (1) Axiom $M$ is valid in the quotient space of any finite $C W$-complex.

(2) Axiom $G$ is valid in the quotient space of a finite $C W$ complex iff each connected component of the $C W$-complex has a unique top cell.

Proof. (1) Let $X$ be any finite CW-complex, and let $c \in$ $Q(X)$ be any point. Then $c$ is in the closure of some point $t$ corresponding to a top cell, so $t$ is open.

Case $1(t \in\|P\|)$. Then $c \in \operatorname{cl}(\{t\})=\operatorname{cl}(\operatorname{int}(\{t\})) \subseteq \operatorname{cl}(\operatorname{int}(\|P\|))$.

Case $2(t \notin\|P\|)$. Then $t \in\|P\|^{C}$, so $c \in \operatorname{cl}\left(\operatorname{int}\left(\|P\|^{C}\right)\right)$.

$(2)(\Leftarrow)$ If $t$ corresponds to a top cell, then its entire connected component is in $\operatorname{int}(\operatorname{cl}(\{t\}))$. Since each connected component of a finite CW-complex contains a top cell and the point in $Q(X)$ corresponding to this top cell is either in $\|P\|$ or in $\|P\|^{C},\|(\square \diamond P) \vee(\square \diamond \neg P)\|=X$.

$(\Rightarrow)$ If a connected component of a finite CW-complex contains at least two top cells, then there two top cells whose closures have nonempty intersection, so the intersection contains at least one cell. Let $t_{1}$ and $t_{2}$ be points in the quotient space corresponding to such two top cells and let $c$ be the point corresponding to a cell in the intersection of their closures. Consider a validation mapping such that $t_{1} \in\|P\|$ and $t_{2} \notin\|P\|$. Since $t_{2}$ corresponds to a top cell, it is not in the boundary of any other point; therefore $t_{2} \notin \mathrm{cl}(\|P\|)$, so $t_{2} \in(\operatorname{cl}(\|P\|))^{C}$. Then $c \in \operatorname{cl}\left(\left\{t_{2}\right\}\right) \subseteq \operatorname{cl}\left((\operatorname{cl}(\|P\|))^{C}\right)$; therefore $c \notin\left(\operatorname{cl}\left((\operatorname{cl}(\|P\|))^{C}\right)\right)^{C}=\|\neg \diamond \neg \diamond P\|=\|\square \diamond P\|$. Since $t_{1} \notin$ $\mathrm{cl}(\|\neg P\|)$, in a similar way, we have $c \notin\|\square \diamond \neg P\|$. Thus $\|(\square \diamond P) \vee$ $(\square \diamond \neg P) \| \neq X$.

\section{Competing Interests}

The authors declare that they have no competing interests.

\section{Acknowledgments}

The authors are very grateful to Fred Cohen for suggesting the study of quotient spaces of CW-complexes and to the College of Science and Mathematics of the California State University, Fresno for supporting this work.

\section{References}

[1] J. C. McKinsey and A. Tarski, "The algebra of topology," Annals of Mathematics. Second Series, vol. 45, pp. 141-191, 1944.

[2] G. Mints, "A completeness proof for propositional S4 in cantor space," in Logic at Work: Essays Dedicated to the Memory of Helena Rasiowa, E. Orlowska, Ed., vol. 24 of Studies in Fuzziness and Soft Computing, pp. 79-88, Physica, Heidelberg, Germany, 1998.

[3] G. Mints and T. Zhang, "A proof of topological completeness for S4 in $(0,1)$, , Annals of Pure and Applied Logic, vol. 133, no. 1-3, pp. 231-245, 2005.

[4] M. Aiello, J. van Benthem, and G. Bezhanishvili, "Reasoning about space: the modal way," Journal of Logic and Computation, vol. 13, no. 6, pp. 889-920, 2003.

[5] G. Bezhanishvili and M. Gehrke, "Completeness of S4 with respect to the real line: revisited," Annals of Pure and Applied Logic, vol. 131, no. 1-3, pp. 287-301, 2005.

[6] L. A. Steen and J. Seebach, Counterexamples in Topology, Springer, New York, NY, USA, 1995.

[7] A. Chagrov and M. Zakharyaschev, Modal Logic, vol. 35 of Oxford Logic Guides, The Clarendon Press, Oxford, UK, 1997. 


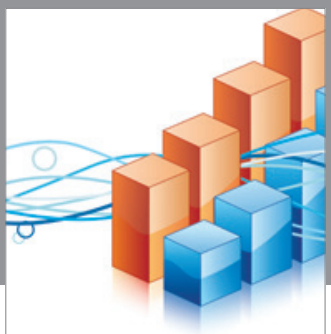

Advances in

Operations Research

vatem alat4

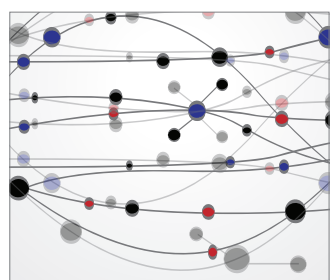

\section{The Scientific} World Journal
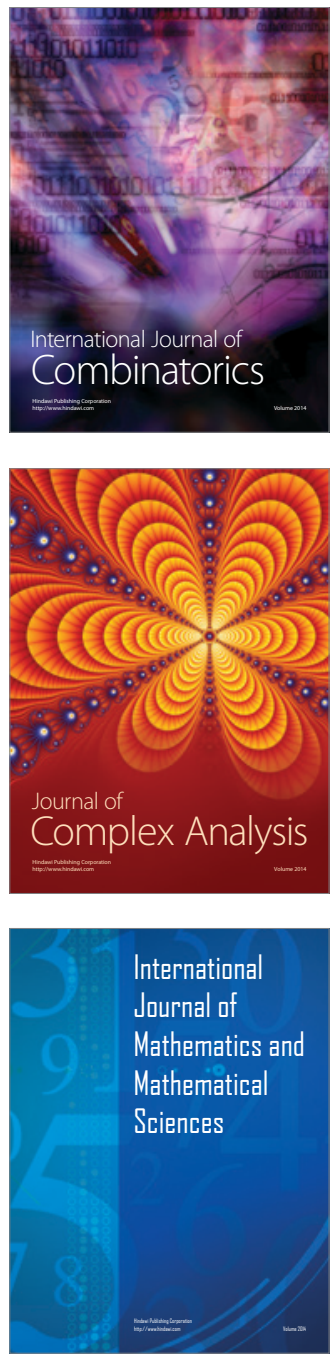
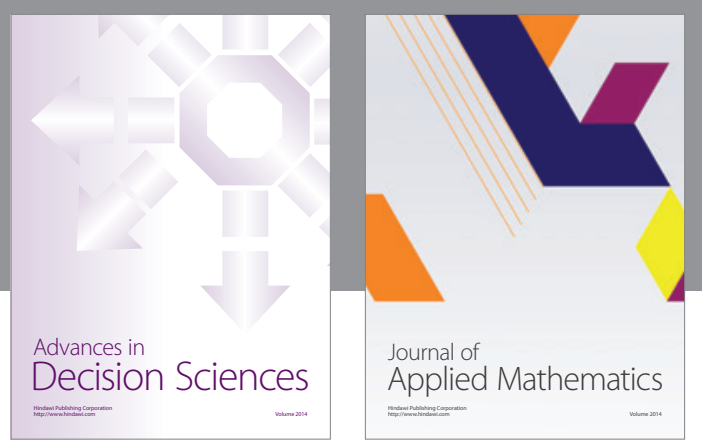

Algebra

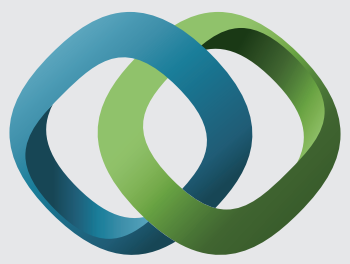

\section{Hindawi}

Submit your manuscripts at

http://www.hindawi.com
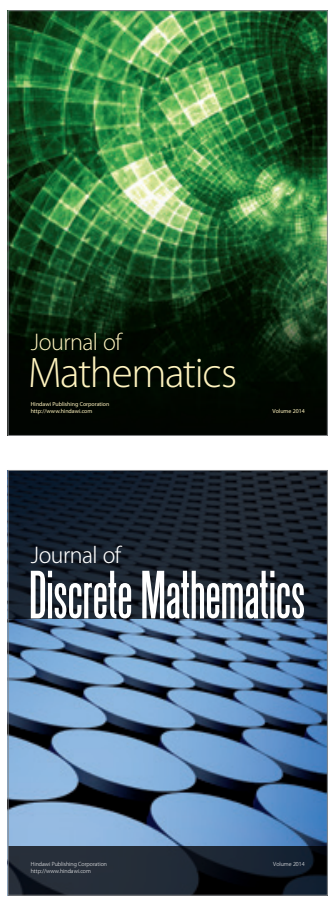

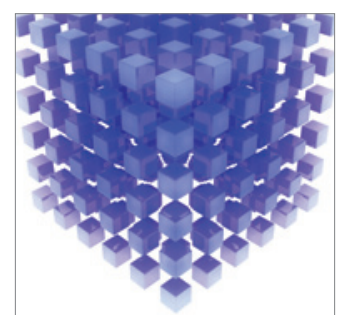

Mathematical Problems in Engineering
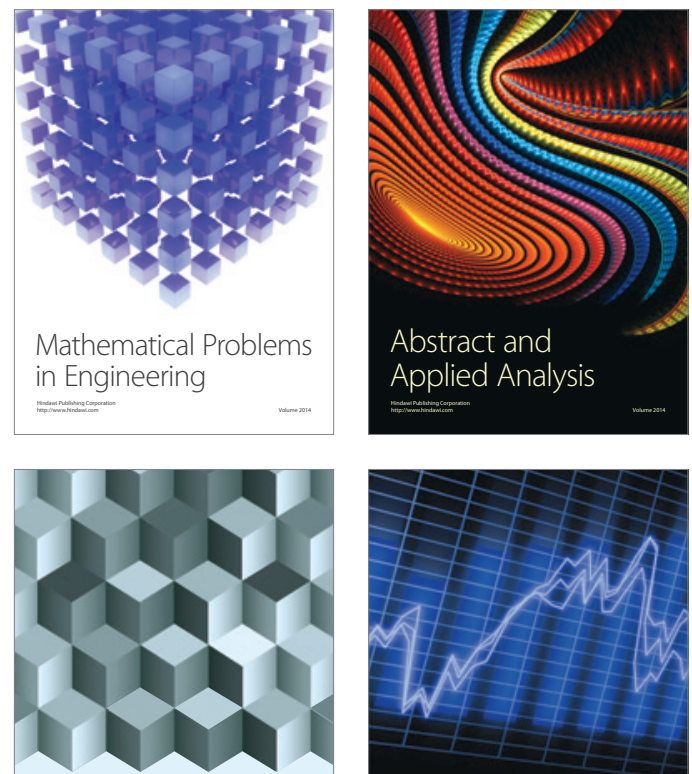

Journal of

Function Spaces

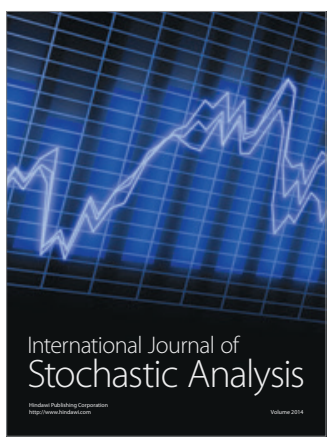

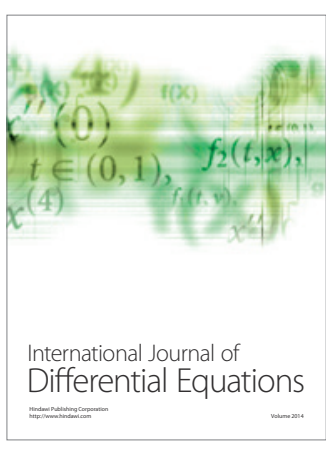
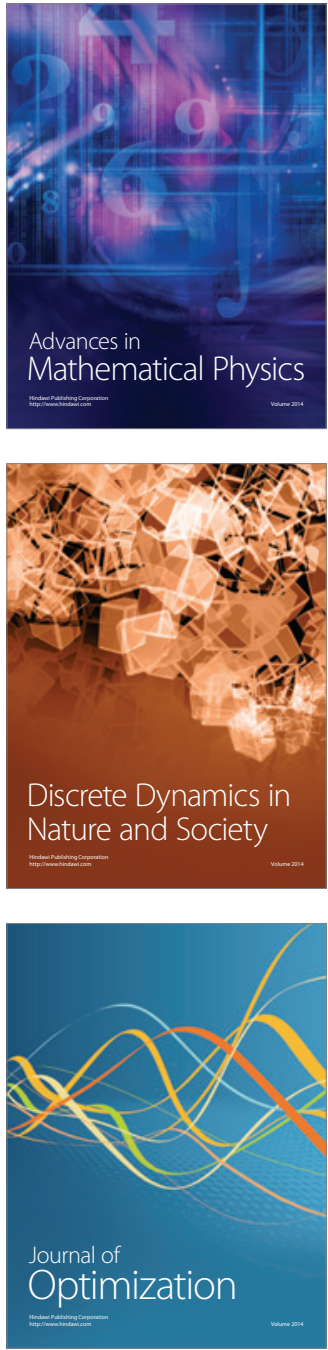